\title{
The effect of surface grooves on film breakdowns in point contacts
}

ŠPERKA, P.; KŘUPKA, I.; HARTL, M.

Tribology International

2016, vol. 102, October 2016, pp. 249-256

ISSN: 0301-679X

DOl: https://doi.org/10.1016/j.triboint.2016.05.031

Accepted manuscript

(C) 2016. This manuscript version is made available under the CC-BY-NC-ND 4.0 license (http://creativecommons.org/licenses/by-nc-nd/4.0/), doi: https://doi.org/ 10.1016/j.triboint.2016.05.031

Final version available from https://www.sciencedirect.com/science/article/pii/S0301679X16301220 


\title{
The Effect of Surface Grooves on Film Breakdowns in Point Contacts
}

\author{
P. Sperka ${ }^{1}$, I. Krupka, M. Hartl \\ Faculty of Mechanical Engineering, \\ Brno University of Technology, \\ Technicka 2896/2, 61669 Brno, Czech Republic
}

\begin{abstract}
Surface roughness plays an important role in transition from full to mixed elastohydrodynamic regime. One kind of features appearing on a real rough surface are grooves longer than contact diameter. It has already been reported that these grooves cause a local film reduction or a complete collapse. In this study, a ball-on-disc optical tribometer was used to quantitatively study the effect of grooves on film thickness in a point contact. It was observed that the main dependence on speed, the so-called lift-off curve, generally follows the logarithm function. The effects of build-up material, load, slide/roll ratio and groove geometry are presented. These results were fitted to the analytical description, which enables the estimation of groove effects on point contact lubrication.
\end{abstract}

\section{KEY WORDS}

elastohydrodynamic lubrication; film thickness; surface roughness; surface texture

\section{INTRODUCTION}

Formation of elastohydrodynamic lubrication (EHL) film between the smooth surfaces has been extensively studied. Nevertheless, surfaces of real mechanical parts have surface roughness. This can be related to the increased friction and enhanced damaging processes, e.g. wear, fatigue, etc. Formation of wear on practical surfaces is a symptom of transition from the full EHL regime to the mixed lubrication regime where the contacts between surface features occur. The main aim of experimental studies is to describe and explain the mechanisms which lead to transition from the EHL regime to the mixed lubrication regime.

Generally, features on rough surfaces could be divided into peaks and valleys. Together, they form a surface topography with a certain height distribution of surface area known as a bearing area curve. This curve is usually interpreted in tribological studies so that the peaks are being worn out at first, while the part of the topography that corresponds to valleys persists on the surface. There is a skewness roughness parameter $R$ sk related to the shape of the bearing area curve. If this parameter is negative, the topography contains more valleys than peeks and vice versa. Some researchers have studied the relation between the skewness parameter of rough surface and tribological properties [1-3]. Nevertheless, this kind of study represents an indirect observation where various effects like material, lubrication effects of complex roughness features, chemistry of tribolayers, etc. could have an unknown contribution to the final result. A more essential approach is to focus on the modelling of mixed lubrication (e.g. [4, 5]) or the modeling of contact problem (e.g. [6]).

Three basic types of surface roughness features, i.e. ridges, dents and grooves, were investigated in experimental studies of roughness effects on lubrication. It was described that dents cause only a local reduction of film thickness when passing the contact inlet; afterwards, a film thickness increase can be observed once they enter the high pressure zone [7,8].

\footnotetext{
${ }^{1}$ Corresponding author. Tel.: +420 541143 323; E-mail address: sperka@fme.vutbr.cz
} 
Additional pressure rippling caused by ridges and bumps lead to their large deformation in an EHL contact that could effectively protect the surfaces against a direct contact [9-11]. The grooves longer than the contact area are connected with a side leakage which causes a local reduction in film thickness or a complete collapse [12]. Therefore the grooves can be regarded as the most dangerous type of roughness features for distribution of lubricant film thickness in a point contact.

Pioneering experimental work on the effects of grooves in EHL contact by using optical interferometry has been done by Wedeven and Cusano [13, 14]. In their papers, deformation of surfaces caused by micro-EHL pressure was studied for perpendicular and parallel orientation of groove under pure rolling and pure sliding conditions. Another extensive study was led by Kaneta $[12,15]$. The effects of surface grooves were studied under various slide/roll ratios $(S R R)$ and mean speeds. It was shown that the side leakage is controlled by the groove width and depth. A groove on a real rough surface was studied by Hartl [16]. It can be stated that this groove causes the same effects as an artificial groove. Ali [17] studied shallow grooves with length less than a diameter of Hertzian contact. It was revealed that this type of groove works as a powerful oil reservoir. Under these conditions, the grooves do not cause a significant decrease in film thickness or a complete film collapse. More complete review on mixed lubricant and roughness effects in EHL contact can be found in $[18,19]$.

There exists a fundamental difference in groove effects on lubrication of conformal contacts [20-22] and non-conformal contacts [15]. In conformal contacts or the contacts between nominally flat surfaces, where pressure and lubricant viscosity are low, surface grooves could have a positive effect. However, this study concentrates on non-conformal contacts of elastohydrodynamic lubrication where a high lubricant viscosity, provided by lubricant piezo-viscous effect, is important to build a film layer and separate surfaces.

As far as the authors know there is no publication that quantitatively reports on the effects of transversely oriented surface grooves on film thickness in a point contact. The aim of this paper is to quantitatively describe the effects of operating conditions and the groove geometry on film thickness distribution.

\section{EXPERIMENTAL APPARATUS AND MATERIALS}

Measurements were conducted using a ball-on-disc optical tribometer. In this apparatus, an EHL point contact was formed between a steel ball and a glass disc. The lower surface of BK7 glass disc is sputtered with a semi-reflecting chromium layer. The steel ball AISI 52100 with a groove has a diameter of $25.4 \mathrm{~mm}$. The elastic modulus of the steel ball is 210 $\mathrm{GPa}$ and that of the glass disc is $81 \mathrm{GPa}$. The reduced elastic modulus is equal to $123.8 \mathrm{GPa}$. Three mineral base oils from group I were used, namely R560/88, SN650, R825/95 having dynamic viscosities of $1.15,0.356,0.06 \mathrm{~Pa} \cdot \mathrm{s}$ at $25{ }^{\circ} \mathrm{C}$, respectively. All experiments presented in this paper were carried out at room temperature of $24.5 \pm 0.5^{\circ} \mathrm{C}$. Lubricant film thickness was evaluated by the colorimetric interferometry technique [23]. In the optical design, a configuration without silica spacer layer on the bottom side of disc was used [24].

Tab. 1 Parameters of grooves

\begin{tabular}{|l|c|c|c|c|}
\hline $\begin{array}{l}\text { Groove } \\
\text { indication }\end{array}$ & $\begin{array}{c}D \\
(\mathrm{~nm})\end{array}$ & $\begin{array}{c}W \\
(\mu \mathrm{m})\end{array}$ & $\begin{array}{c}H_{B} \\
(\mathrm{~nm})\end{array}$ & \\
\hline K1 & 830 & 8 & 0 & \\
\hline K2 & 1140 & 10 & 0 \\
\hline K3 & 1790 & 12 & 0 \\
\hline R1 & 810 & 50 & 0 \\
\hline R2 & 280 & 40 & 0 \\
\hline R3 & 620 & 52 & 0 \\
\hline R4 & 1370 & 63 & 0 \\
\hline R5 & 930 & 54 & 270 \\
\hline
\end{tabular}




\begin{tabular}{|l|c|c|c|c|}
\hline R6 & 630 & 53 & 0 & \multirow{2}{*}{} \\
\cline { 1 - 3 } RS1 & 80 & 31 & 0 & \\
\cline { 1 - 3 } RS2 & 240 & 42 & 0 & \\
\cline { 1 - 3 } RS3 & 540 & 49 & 0 & \\
\cline { 1 - 3 } RS4 & 940 & 56 & 0 & \\
\hline
\end{tabular}

Grooves were produced by means of indentation rig which uses an electromagnetic solenoid to create the impact between the indenter tip and the ball surface. This device uses a stepper motor to create a linear motion and the indentation process is fully automated as it is described in [17]. In this paper, three types of indenter tips were used. Two of them were Rockwell indenters with a tip angle of $120^{\circ}$ and a slightly different radius of tip within the range of $0.2 \pm 0.01 \mathrm{~mm}$. The other indenter was the Knoop indenter with a tip of tetrahedral diamond pyramid and tip angles of 172.5 and $130^{\circ}$ in two perpendicular planes. It was decided to primarily test the groove geometry without build-up material which is created during production due to plastic deformation. Therefore, a post-production treatment was needed to remove the build-up material after indentation. This material was removed by polishing with diamond paste. The polishing process did not significantly change the width of the groove but it significantly reduced its depth. Practically, a total depth of one groove before polishing was $930 \mathrm{~nm}$ (see the groove R5 in Tab. 1) and the groove depth after polishing was $630 \mathrm{~nm}$ (see the groove R6 in Tab. 1). Altogether, thirteen different grooves were studied. Twelve of them were polished to remove buildup material and one was left unpolished (R5). Data of grooves are listed in Tab. 1. The grooves K were produced using the Knoop indenter while the grooves R, RS used Rockwell indenters. A commercial optical profilometer was used to determine the grooves geometry. Grooves were characterized by depth $D$, width $W$ and height of build-up material $H_{B}$. These geometry parameters were schematically shown in Tab. 1. Representative profiles of unpolished groove (R5), polished groove (RS3) and the grove created by Knoop indenter (K3) are shown in Fig. 1. Because grooves had rounded edges, the width of the groove was determined as a distance between imaginary intersections of the straight parts of groove arms with smooth surface geometry.

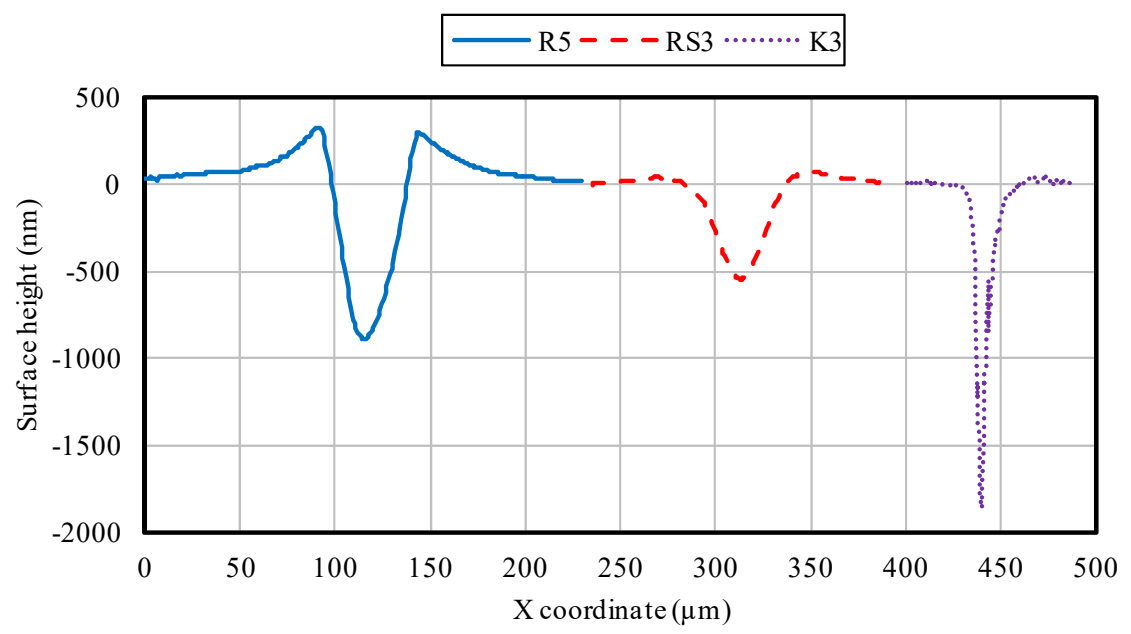

Fig. 1 Profiles of grooves R5, RS3 and K3

\section{RESULTS AND DISCUSSION}

Fig. 2 shows interferograms observed at various time periods of the passage of groove K1 through the EHL point contact under conditions of mean speed of $0.02 \mathrm{~m} \cdot \mathrm{s}^{-1}$, Hertzian pressure $0.54 \mathrm{GPa}$, SN650 oil, slide/roll ratio $S R R=1$ (Fig. $2 \mathrm{a}$-e) and $S R R=-1$ (Fig. 2f-j). Slide/roll ratio is defined as $2\left(u_{\mathrm{d}}-u_{\mathrm{b}}\right) /\left(u_{\mathrm{d}}+u_{\mathrm{b}}\right)$, where $u_{\mathrm{d}}$ and $u_{\mathrm{b}}$ are speeds of disc and ball respectively. In this figure, the contact inlet is on the left side. As it has already been described by Kaneta[12, 15] when the leading edge of the groove enters the contact region (Fig. 2a,f), there is a rapid pressure drop inside the groove. Due to this pressure drop, the viscosity of lubricant is not sufficient to allow a flow continuation in entrainment direction. 
Lubricant is trapped by the groove, with the length greater than the diameter of Hertzian contact and lubricant is drained to the sides of the groove. The side leakage causes a local reduction or a complete collapse of film thickness upstream of entrainment motion. This local reduction in film thickness moves at mean speed across the entire contact region (Fig. $2 b-$ $d, g-i)$. It means that when the speed of surface with the groove (ball) is faster than the speed of lubricant $(S R R<0)$, this local reduction takes place behind the groove (upstream from the groove). On the contrary, when the speed of surface with the groove (ball) is slower than the speed of lubricant $(S R R>0)$, the local reduction of film thickness is takes place in front of the groove (downstream from the groove). A full EHL film is formed when the groove passes through the contact area (Fig. 2e,j).

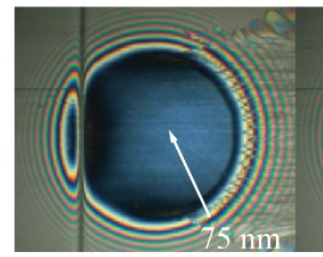

a)

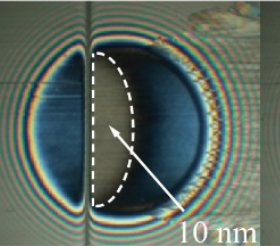

b)

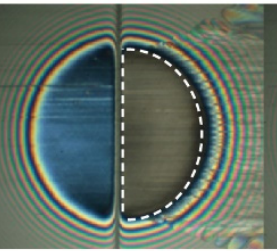

c)

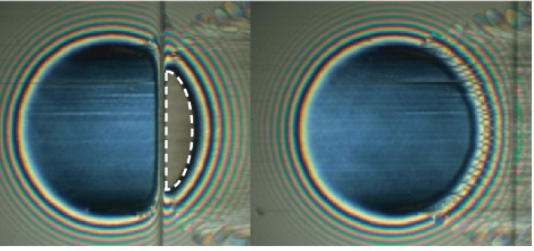

d) e)

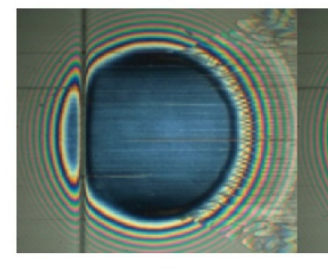

f)

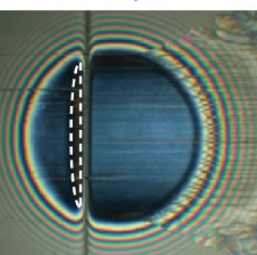

g)

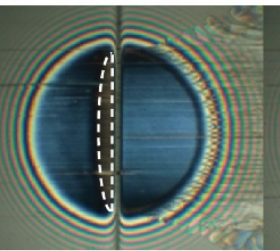

h)

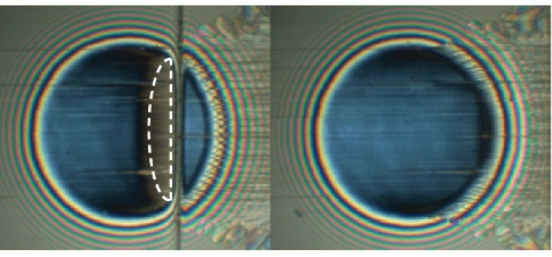

i)

j)

Fig.2 Groove K1 at various positions in the contact area under SRR: a-e) 1, f-j) -1; speed $0.02 \mathrm{~m} . \mathrm{s}^{-1}$ and Hertzian pressure $0.54 \mathrm{GPa}$. The area of film thickness influenced by the groove is marked with a white dashed line.

Fig. 2 shows the case when the film decrease continues during the entire passage through the contact area. It is because the side flow by the groove continues during the whole contact, and the lubricant outflow from the groove in the entrainment direction is not recovered by the increasing pressure inside the contact. This can be considered as a film breakdown. Fig. 3 shows a series of interferograms for the same groove and a higher speed. It can be seen that a significant film decrease is generated once the groove reaches the contact inlet (Fig. 3a). After that, inside a highly loaded zone, the lubricant outflow from the groove is recovered and a significant film decrease can be observed only close to the sides (Fig. 3d). The plots in Figs. 4, 6-9 show the conditions for the same behavior as that in Fig. 2 marked with points with black edges; otherwise the behavior is the same as that in Fig. 3.

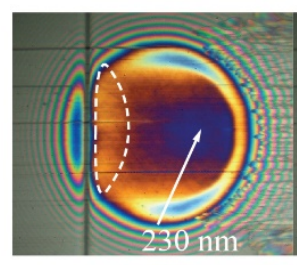

a)

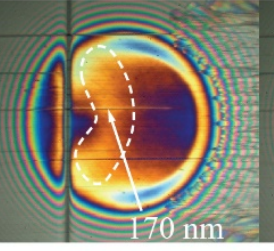

b)

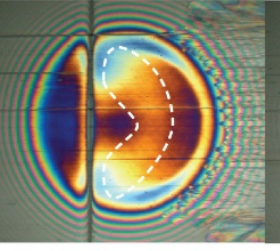

c)

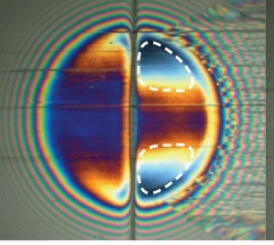

d)

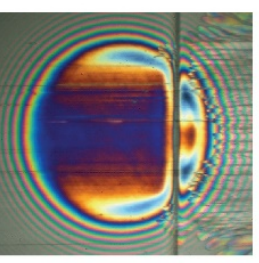

e)

Fig. 3 Groove K1 at various positions in the contact area under $S R R$, speed of $0.08 \mathrm{~m} . \mathrm{s}^{-1}$ and Hertzian pressure 0.54 $\mathrm{GPa}$. The area of film thickness significantly influenced by the groove is marked with white dashed line.

To quantitatively study the effects of grooves on film thickness decrease, it is necessary to define the parameter that will express the influence on in-contact film thickness. A new parameter $R_{G I}$ (groove influence ratio) was suggested as a ratio of minimum film thickness influenced by the groove in centerline $\left(h_{\text {GImin }}\right)$ divided by central film thickness of smooth surface steady state conditions $\left(h_{\text {smooth }}\right)$. The value of $h_{\text {GImin }}$ was also evaluated in central zone of the contact (out of the outlet constriction). Because, the aim was to capture groove effect in highly loaded zone where thin film thickness can have significant effect on wear and other failures. Groove influence ratio is given by 
The minimum value $h_{\text {GImin }}$ was taken in a moment when groove was approximately in the middle of a contact (Fig. $2 \mathrm{c}$, 2h) for cases when the behavior was considered as the film breakdown (Fig. 2). In cases of behavior with recovery (Fig. 3 ) and $S R R>0$, the value was taken in a moment when groove was approx. in one third of the contact length (Fig. 3c) and the minimum film thickness takes place in approx. two thirds of the contact length (depending on magnitude of $S R R$ ). In both cases the minimum value does not further decrease within central zone and centerline. Nevertheless, evaluated minimum film thickness $h_{\text {GImin }}$ is often not the global minimum film thickness. The global minimum usually occurs on the sides of EHL contact in area where global minimum of smooth contact takes place (horse shoe constriction).

\subsection{Lift-off curve}

To express the effect of the mean speed, measurements with groove R3 were carried out for Hertzian pressure of 0.54 $\mathrm{GPa}, \mathrm{SN650}$ lubricant and slide/roll ratio $S R R=1$. The mean speed was varied to cover a wide range of groove influence ratio. This ratio as a function of mean speed is plotted in Fig. 4. In literature this kind of plot is usually called a "lift-off" curve [25]. This curve means the transition from conditions when groove influence on film thickness is large to the conditions where groove influence disappears. Since $x$ axis is in log scale, it can be concluded that the "lift-off" transition has a logarithmic character.

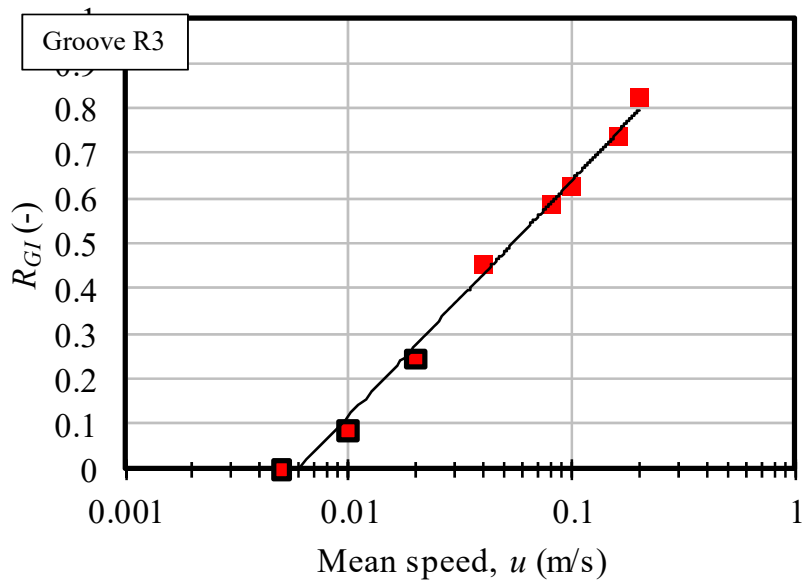

Fig.4 The influence of mean speed on groove influence ratio for groove R3

Interferograms corresponding to the data in Fig. 3 are shown in Fig. 5. A complete collapse of lubricant film thickness $\left(R_{G I}=0\right)$ can be seen at low mean speeds. If the mean speed increases, the lubricant film thickness influenced by the groove increases; hence, the groove influence ratio increases. The value of groove influence ratio $R_{G I}=1$ indicates that the groove has no influence on the distribution of lubricant film thickness in the point contact. It has to be noted that the conditions of $R_{G I}$ close to 1 are hard to obtain and in this case the points in Fig. 4 will deviate from the logarithmic function. 


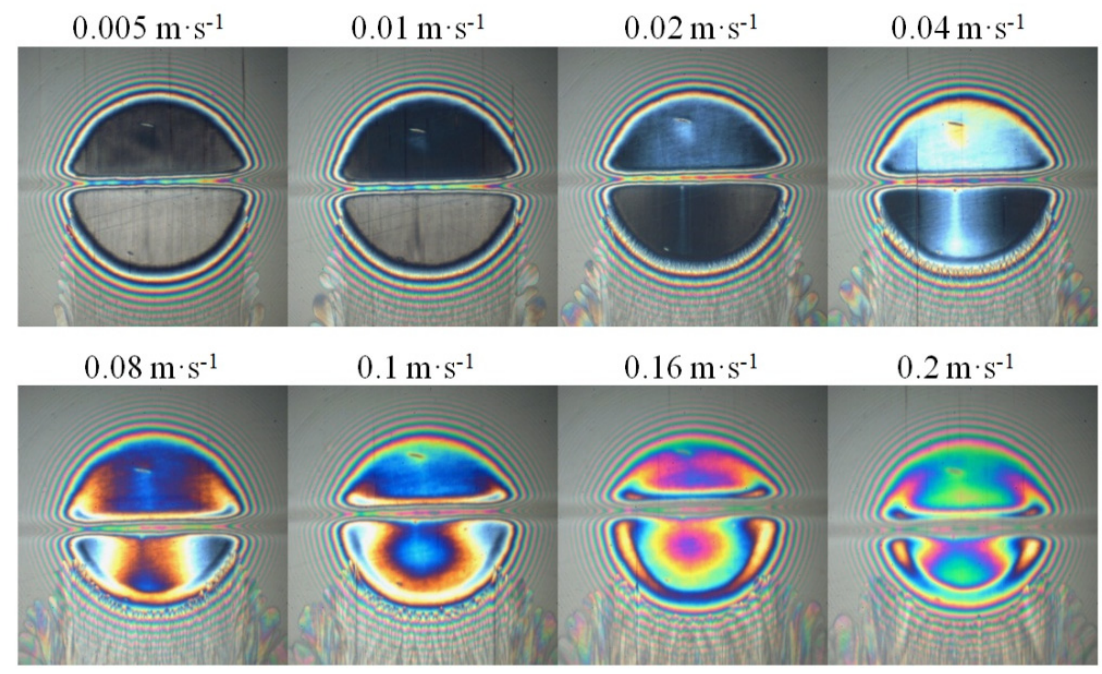

Fig.5 Interferograms of groove R3 in the contact area under various mean speeds

\subsection{Effect of built-up material}

The effect of built-up material has been studied in this paper with the groove R5, SN650 lubricant and conditions of Hertzian pressure of $0.54 \mathrm{GPa}$ and $S R R=1$. After this experiment, the groove R5 was polished to remove the built-up material. The groove created after polishing of R5 was labeled as the groove R6. Experiments under the same conditions as for the groove R5 were carried out for the groove R6. The results of these experiments are presented in Fig. 6 where the plot clearly shows that the influence of build-up material on lubricant film thickness in the point contact is significant. Film thickness influenced by the groove R5 is held approximately at the value $R_{G I}=0.1$ for speeds from 0.02 to $0.16 \mathrm{~m} / \mathrm{s}$. More important is that the build-up material moves the points in the figure to the right side towards higher speeds. Also the film thickness recovery is considerably steeper than that of the groove without build-up material. It has to be noted that the groove R5 has 50\% larger depth than the groove R6, so the shift in speeds will be partially due to a different depth. Generally, it can be stated that the presence of built-up material contributes to film breakdown, so it has a negative effect.

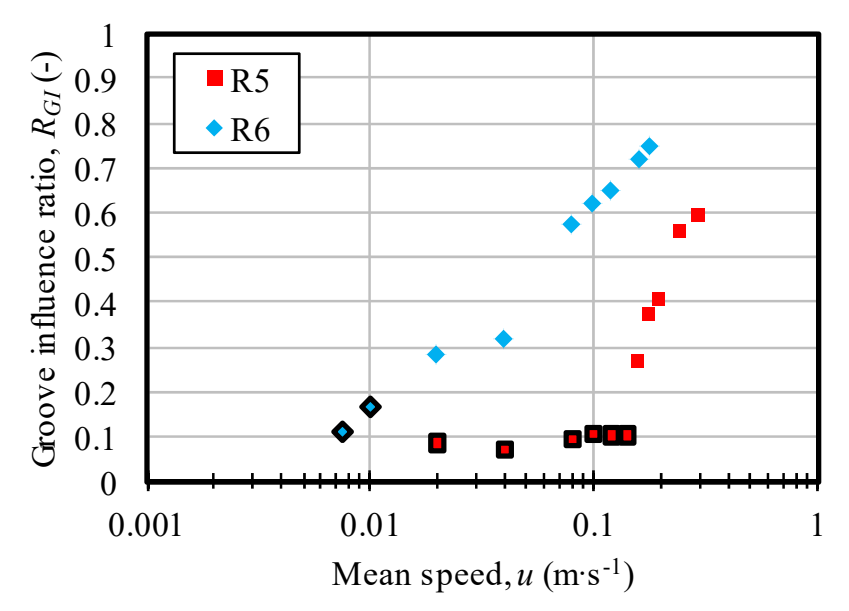

Fig.6 The effect of build-up material on "lift-off" curve

\subsection{Effect of slide/roll ratio}

The effect of $S R R$ was tested by carrying out experiments for the groove R1, Hertzian pressure of 0.6 GPa, SN650 lubricant and $S R R=0.5,1,1.5,-0.5,-1$ and -1.5 . The results of these experiments are shown in Fig. 7 for four different mean speeds. It can be seen that the groove influence ratio is considerably lower for positive values of $S R R$. Accordingly, the groove affects film thickness more significantly for the case of faster disc than for the case of faster ball. Despite the 
same magnitude of sliding, it could be suggested that the case of $S R R<0$ (faster surface with groove) leads to a higher hydrodynamic effect inside the groove resulting in earlier flow continuation in entrainment direction. A difference between the speed of lubricant and the speed of surface with groove (ball) also determines the edge of the groove through which lubricant flows into the contact area as it was described by Kaneta [12, 15]. This can be seen in interferograms in Fig. 2. From Fig. 7 it can also be seen that the magnitude of sliding has a negligible effect on the groove influence ratio.

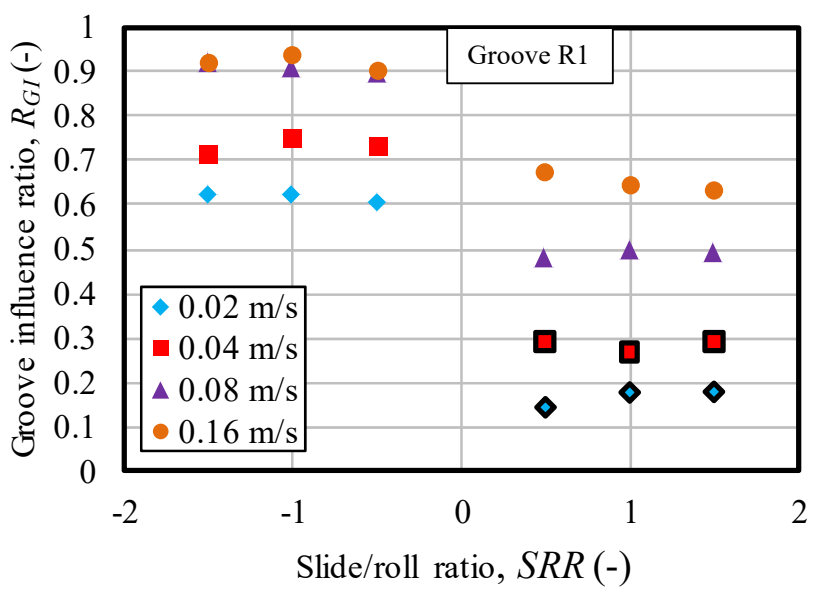

Fig.7 The effect of slide/roll ratio on groove influence ratio

\subsection{Effect of contact pressure}

The groove influence ratio as a function of mean speed for three different values of Hertzian pressure is plotted in Fig. 8. The experiments were carried out for the groove R3, slide/roll ratio $S R R=1$ and mineral oil SN650. Fig. 8 shows that the measurement results for all three values of Hertzian pressure can be described by logarithmic function. The value of groove influence ratio for these three pressures is nearly the same at a given mean speed. A small deviation of measured points occurs at lower mean speeds where the groove causes a greater decrease of lubricant film thickness in the contact area. It can be stated that in this pressure range the pressure change does not influence the distribution of lubricant film thickness when the groove passes through the contact area. It is necessary to mention that this pressure range is the maximum possible when using this type of tribometer and this configuration of contact pair (glass disc, steel ball). Higher contact pressure (up to $1.2 \mathrm{GPa}$ ) could be achieved by using a sapphire disc. However, experiments with sapphire disc will add another variable, i.e. the effect of elastic modulus. This effect could be expressed in prospective study using a wider range of material combinations.

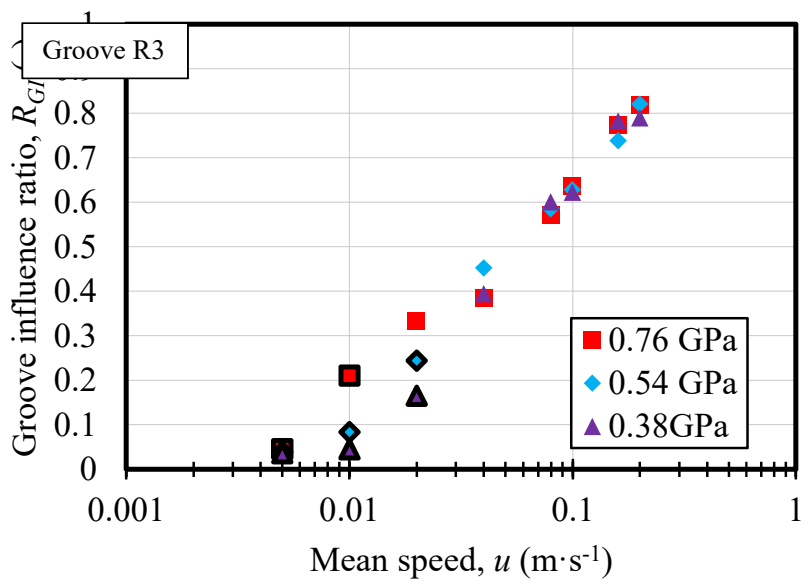

Fig.8 The influence of mean speed on groove influence ratio for three Hertzian pressures 


\subsection{Effect of viscosity}

Three various mineral oils were used to evaluate the effect of viscosity. The parameters of these oils are described in Section 2. Experiments were carried out for groove R3, slide/roll ratio $S R R=1$ and Hertzian pressure 0.54 GPa. The results are plotted against a product of mean speed and viscosity in Fig. 9. It was observed that if the viscosity of lubricant decreases, a higher mean speed is necessary to obtain the same $R_{G I}$ and vice versa. On this basis it can be stated that increasing the mean speed and viscosity has the same effect on the lubrication process. These experiments express mainly the effect of lubricant viscosity but they do not describe the influence of pressure-viscosity coefficient. The used oils are similar mineral oils from group 1 where approximately the same pressure-viscosity coefficient can be expected (approximately in the range of 20 to $25 \mathrm{GPa}^{-1}$ at $25^{\circ} \mathrm{C}$ ). Exact effects of pressure-viscosity coefficient can be studied using other lubricants (e.g. glycerol, polyalphaolefin) or carrying out experiments at various temperatures when the pressureviscosity coefficient decreases with increasing temperature.

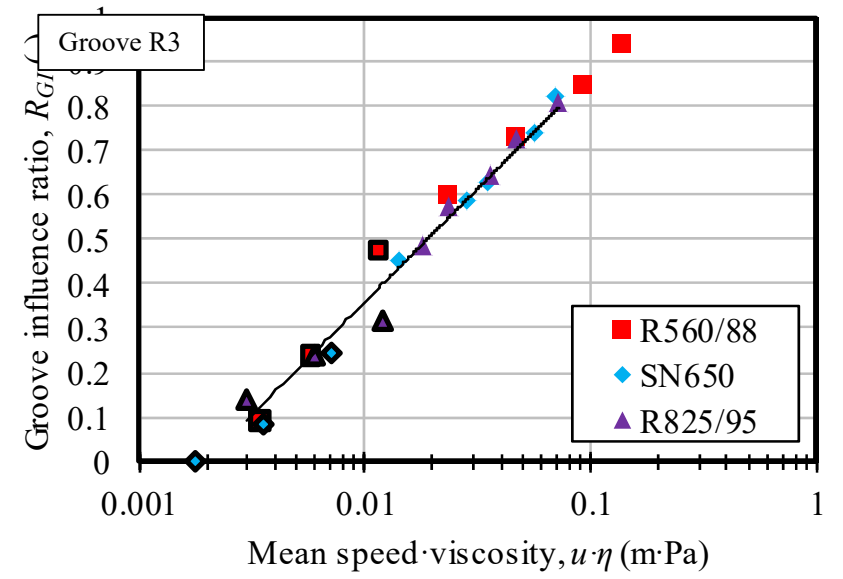

Fig.9 The influence of product of mean speed and viscosity on groove influence ratio for three mineral oils

\subsection{Effect of groove geometry}

A set of 10 grooves was created by using three different indenters. This set covers a range of width from 8 to $63 \mu \mathrm{m}$ and a range of depth from 80 to $1790 \mathrm{~nm}$. All parameters of tested grooves are listed in Tab. 1. All investigated grooves in the contact area under the conditions of mean speed of $0.04 \mathrm{~m} \cdot \mathrm{s}^{-1}$ are shown on interferograms in Fig. 10.

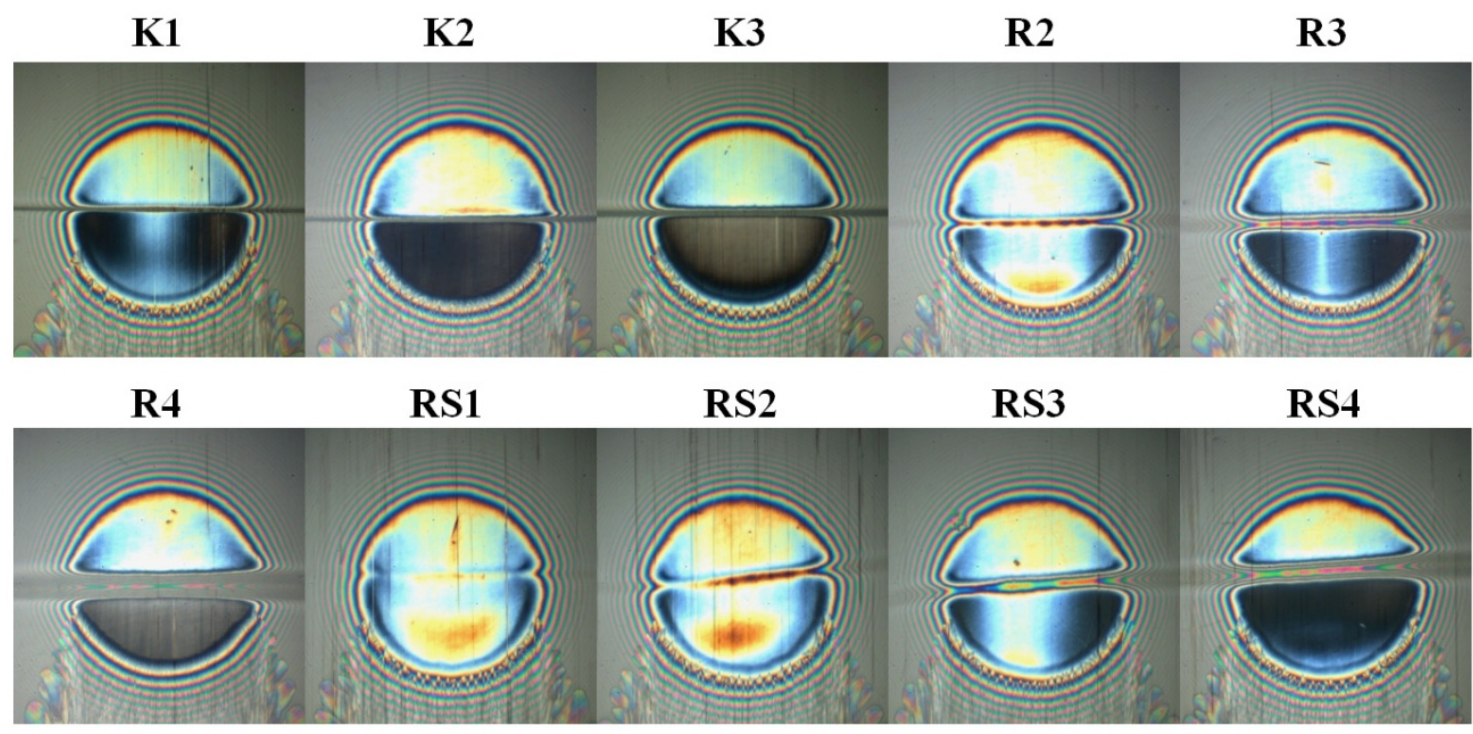

Fig.10 All investigated grooves in contact area (mean speed $0.04 \mathrm{~m} \cdot \mathrm{s}^{-1}$ ) 
Experiments were carried out under the same operating conditions of Hertzian pressure ( $0.54 \mathrm{GPa}$ ), temperature of 24.5 $\pm 0.5{ }^{\circ} \mathrm{C}, S R R=1$ and mineral oil SN 650 . In these experiments, only the mean speed varied to create the so-called "liftoff' curve. From all experiments it was observed that the transition from a large groove influence on film thickness to a disappearing groove influence can be described by a logarithmic function. The individual "lift-off" curves differ by central point, i.e. speed at 50\% groove influence, and the slope of the curve. In this paper the center of the logarithmic transition was chosen as a basic parameter for assessment of groove geometry. The central point corresponds to the mean speed at which the groove influence ratio is 0.5 designated as $u_{\text {Rgiso }}$.

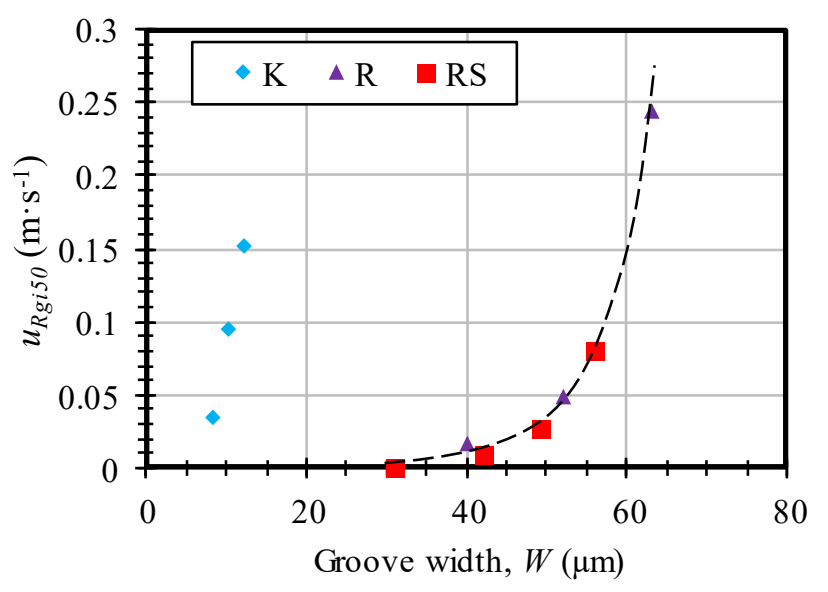

Fig. 11 The influence of groove width on $u_{\text {Rgiso }}$

Fig. 11 and Fig. 12 show the influences of groove width and depth on $u_{\text {Rgi50, }}$, respectively. In these plots, the grooves are distinguished by an indenter (K, R and RS) used for their production. From these plots it is clear that if the groove width or depth decrease then $u_{\text {Rgi } 50}$ decreases. It means that a lower mean speed is required to obtain $50 \%$ of the groove influence ratio. For the groove width of approx. $30 \mu \mathrm{m}$ and depth of approx. $100 \mathrm{~nm}$, the $u_{\text {Rgiso }}$ is zero; therefore, a groove of these parameters never influences the lubricant film thickness up to $50 \%$ of film thickness for smooth surface. From Fig. 11 and Fig. 12, it can be seen that the distribution of points produced by the indenters R and RS is approximately equal, but the points for indenter $\mathrm{K}$ are significantly different. This difference is due to a specific characteristic of geometry produced by various indenters like the proportionality between depth and width of grooves. The grooves formed by indenters $\mathrm{R}$ and RS have approximately the same ratio of depth and width. Conversely, the grooves formed by indenter K have a different ratio since they are narrow and deep.

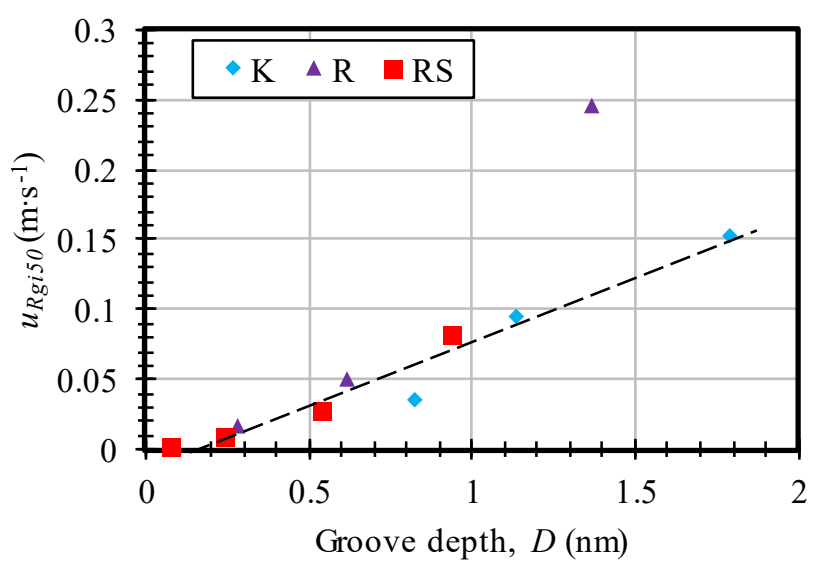

Fig.12 The influence of groove depth on $u_{\text {Rgi50 }}$

It seems that the data points in Fig. 11 and Fig. 12 follow certain trends. Generally, the width and depth have a joined effect on speed $u_{\text {Rgi50}}$; therefore, it is difficult to extract the individual effects of width and depth. Nevertheless, since the 
$\mathrm{K}$ grooves have a very similar width but different depth and simultaneously the data for K grooves in Fig. 12 follow most data points of other two indenters, it can be suggested that the dependency of speed $u_{\text {Rgiso }}$ on depth is close to or directly linear. Simultaneously, the dependency of groove width on speed $u_{\text {Rgiso }}$ has a trend of higher power than the linear function. One point out of the linear trend in Fig. 12 is due to large influence of groove width visible in Fig. 11 (the most right point). Possibly, points for K grooves in Fig. 11 do not follow the other points due to their large depth. It can be concluded that, the effect of groove width has a much stronger impact on film thickness than the groove depth.

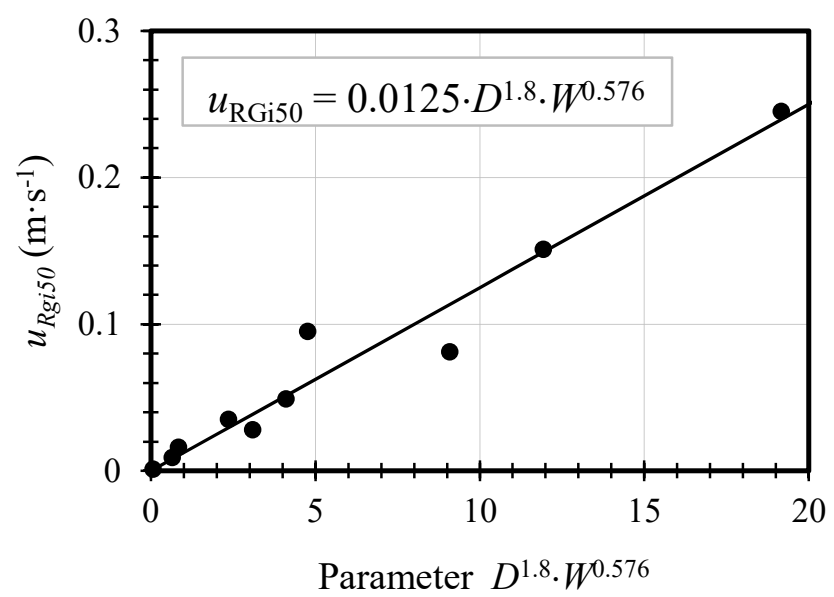

Fig. 13 The influence of groove geometry parameter on parameter $u_{R g i 50}$

The data from Fig. 11, 12 can be fitted by a single line based on combined geometry parameter

$u_{R g i 50}=0.0125 D^{1.8} \cdot W^{0.576}$

where groove depth $D$ and groove width $W$ are in $\mu \mathrm{m}$. The plot is shown in Fig. 13. It enables a prediction of speed with $50 \%$ film reduction caused by a groove of certain geometry. Note that this data corresponds to a point contact of steel on glass and the groove longer than the contact width oriented perpendicular to the entrainment direction and conditions of $S R R=1$.

It has already been mentioned that practically a complete "lift-off" curve follows a logarithm function and this function can be described by the central point; in this case represented by $u_{\text {Rgiso }}$ speed, and the slope of the curve. For ten studied grooves, it was possible to adequately fit the logarithm slope by quadratic function based on groove depth, see Fig. 14 .

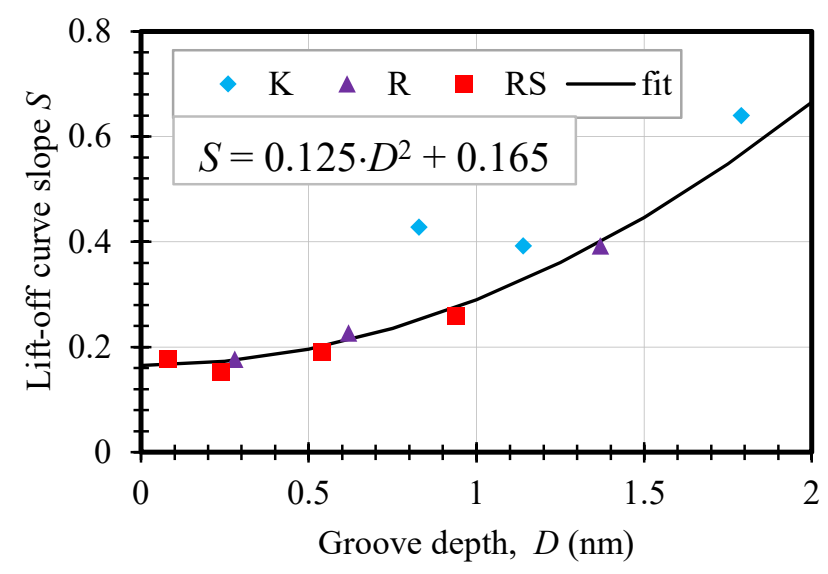

Fig. 14 Dependence of "lift-off" curve slope on groove depth

After that, the complete lift-off curve can be analytically described by a simple equation

$R_{G I}=S\left[\ln (u)-\ln \left(u_{R g i 50}\right)\right]+0.5$

$S=0.125 D^{2}+0.165$ 
The $\mathrm{R}_{\mathrm{GI}}$ is defined on the interval 0 to 1 ; therefore, negative values has to be truncated to zero and values over 1 truncated to 1 . Equation 3 can be used to predict a film thickness reduction produced by groove of known width and depth for the given speed. In the ongoing study, it would be worth to quantify the effect of material elasticity, inclination angle of the groove and lubricant piezoviscosity.

\section{CONCLUSION}

The effects of transversely oriented grooves on distribution of film thickness in an EHL point contact have been studied in this paper. A ball-on-disc optical tribometer was used to measure the minimum film thickness influenced by groove and the central film thickness for smooth surface. Lubricant film thickness was evaluated by thin film colorimetric interferometry. Three types of indenters were employed to produce various geometries of groove. The effects of groove geometry, viscosity of lubricant, slide/roll ratio, mean speed, and contact pressure were studied in this paper.

The effects of these variables were evaluated based on suggested parameter $R_{G I}$ (groove influence ratio). The groove influence ratio is defined as film thickness influenced by the groove divided by film thickness for smooth surface. Significance of this study can be concluded by the following points:

- If the mean speed increases, then the lubricant film thickness influenced by the groove increases, and hence the groove influence ratio $R_{G I}$ increases.

- Transition between a large groove influence on film thickness and a disappearing groove influence can be described by a logarithmic function - the so-called "lift-off" curve.

- Change of contact pressure $(0.38$ to $0.76 \mathrm{GPa})$ does not affect distribution of lubricant film thickness when the groove passes through the contact area.

- 'Lift-off" curves for mineral oils of different viscosity can be unified to master the curve based on the parameter defined as the product of mean speed and dynamic viscosity.

- The effect of groove width and depth was studied for mean speed at which the groove influence ratio is 0.5 , labeled as $u_{\text {Rgi5o. }}$. It can be concluded that the width of groove has a much stronger impact on the speed $u_{\text {Rgi50, }}$, hence also on film thickness, than the depth of the groove.

- An analytical expression for "lift-off" curve was presented based on the central point $u_{\text {Rgiso }}$ and the slope S. A single curve was fitted to the data representing a groove geometry effect. It enables a prediction of film reduction caused by a groove of various geometries.

\section{ACKNOWLEDGEMENTS}

Authors would like to express their thanks to K. Matousek for preparation and carrying out the experiments for this paper. Research leading to these results has received funding from the Czech Science Foundation (grant No. P101/11/1115) and the Ministry of Education, Youth and Sports under the National Sustainability Program I (Project LO1202).

\section{REFERENCES}

[1] Jeng YR. Impact of plateaued surfaces on tribological performance. Tribology Transactions 1996; 39:354-361.

[2] Sedlaček M, Podgornik B, Vižintin J. Influence of surface preparation on roughness parameters, friction and wear. Wear 2009; 266:482-487.

[3] Sedlaček M, Podgornik B, Vižintin J. Correlation between standard roughness parameters skewness and kurtosis and tribological behaviour of contact surfaces. Tribology International 2012; 48:102-112.

[4] Kang YS, Hager CH, Evans RD. Effects of Skewed Surface Textures on Lubricant Film Thickness and Traction. Tribology Transactions 2015; 58:397-406.

[5] Wang WZ, Chen H, Hu YZ, Wang H. Effect of surface roughness parameters on mixed lubrication characteristics. Tribology international 2006; 39:522-527. 
[6] Ghosh A, Sadeghi F. A novel approach to model effects of surface roughness parameters on wear. Wear 2015; 338:73-94.

[7] Kaneta M, Sakai T, Nishikawa H. Optical Interferometric Observations of the Effects of a Bump on Point Contact EHL. Journal of Tribology-Transactions of the ASME 1992; 114:779-784.

[8] Křupka I, Hartl M. The effect of surface texturing on thin EHD lubrication films. Tribology International 2007; 40:1100-1110.

[9] Félix-Quiñonez A, Ehret P, Summers JL. New experimental results of a single ridge passing through an EHL conjunction. Journal of Tribology 2003; 125:252-259.

[10] Choo JW, Olver AV, Spikes HA. The influence of transverse roughness in thin film, mixed elastohydrodynamic lubrication. Tribology International 2007; 40:220-232.

[11] Felix-Quinonez A, Ehret P, Summers JL. On three-dimensional flat-top defects passing through an EHL point contact: a comparison of modeling with experiments. Journal of Tribology 2005; 127:51-60.

[12] Kaneta M. Effects of Surface-Roughness in Elastohydrodynamic Lubrication. JSME International Journal, Series III-Vibration, Control engineering, Engineering for industry 1992; 35:535-546.

[13] Wedeven LD, Cusano C. Elastohydrodynamic Film Thickness Measurements of Artificially Produced Surface Dents and Grooves. ASLE Transactions 1978; 22:369-381.

[14] Cusano C, Wedeven LD. Elastohydrodynamic Film Thickness Measurements of Artificially Produced Nonsmooth Surfaces. ASLE Transactions 1979; 24:1-14.

[15] Kaneta M, Nishikawa H. Local reduction in thickness of point contact ehl films caused by a tranversly oriented moving groove and its recovery. ASME Journal of Tribology 1994; 116:635-639.

[16] Hartl M, Křupka I, Fuis V, Liška M. Experimental study of lubricant film thickness behavior in the vicinity of real asperities passing through lubricated contact. Tribology Transactions 2004; 47:376-385.

[17] Ali F, Kaneta M, Křupka I, Hartl M. Experimental and numerical investigation on the behavior of transverse limited microgrooves in EHL point contacts. Tribology International 2015; 84:81-89.

[18] Spikes HA. Mixed lubrication —an overview. Lubrication Science 1997; 9:221-253.

[19] Morales-Espejel GE. Surface roughness effects in elastohydrodynamic lubrication: A review with contributions. Proceedings of the Institution of Mechanical Engineers, Part J: Journal of Engineering Tribology 2014; 228:12171242.

[20] Siripuram RB, Stephens LS. Effect of deterministic asperity geometry on hydrodynamic lubrication. ASME J. Tribol. 2004; 126:527-534.

[21] Kovalchenko A, Ajayi O, Erdemir A, Fenske G, Etsion I. The Effect of Laser Texturing of Steel Surfaces and SpeedLoad Parameters on the Transition of Lubrication Regime from Boundary to Hydrodynamic. Tribology Transactions 2004; 47:299-307.

[22] Chang L, Jeng YR. Effects of negative skewness of surface roughness on the contact and lubrication of nominally flat metallic surfaces. Proceedings of the Institution of Mechanical Engineers, Part J: Journal of Engineering Tribology 2013; 227:559-569.

[23] Hartl M, Křupka I, Liška M. Elastohydrodynamic film thickness mapping by computer differential colorimetry. Tribology transactions 1999; 42:361-368.

[24] Hartl M, Krupka I, Fuis V, Liska M. Experimental study of lubricant film thickness behavior in the vicinity of real asperities passing through lubricated contact. Tribol Trans 2004; 47: 376-385.

[25] Masen MA, Venner CH, Lugt PM, Tripp JH. Effects of surface micro-geometry on the lift-off speed of an EHL contact. Tribology Transactions 2002; 45:21-30. 